ECCOMAS

\section{Proceedia}

COMPDYN 2021

$8^{\text {th }}$ ECCOMAS Thematic Conference on Computational Methods in Structural Dynamics and Earthquake Engineering Streamed from Athens, Greece, 28 - 30 June 2021

\title{
OPTIMUM DAMPING OF SLENDER MONOPOLE TOWERS BY GYROSCOPIC STABILIZER
}

\author{
Gian Felice Giaccu ${ }^{1}$ Luigi Fenu $^{2}$ Bruno Briseghella $^{3}$ Camillo Nuti $^{3,4}$ \\ ${ }^{1}$ Department of Architecture, Design and Urban Planning, University of Sassari, Alghero, Italy \\ e-mail: gf.giaccu@uniss.it \\ ${ }^{2}$ Department of Civil and Environmental Engineering, and Architecture, University of Cagliari, Ca- \\ gliari, Italy \\ e-mail: 1fenu@unica.it \\ ${ }^{3}$ College of Civil Engineering, Fuzhou University, Fuzhou, Fujian Province, China \\ e-mail: bruno@fzu.edu.cn \\ ${ }^{4}$ Department of Architecture, Roma Tre University, Rome, Italy \\ e-mail: camillo.nuti@uniroma3.it
}

\begin{abstract}
Slender structures as lighting poles and telecommunication towers are usually very sensitive to wind effects due their dynamic characteristics and due to their notable exposure to wind forces. Wind-induced forces produce fluctuating stress, which, despite the assessment of dynamic properties and the study of the environmental conditions, can lead to damage accumulation and consequent collapse of these structures. Careful attention to wind forces has, therefore, to be paid for these typologies of structures, since several damages have been attributed to fatigue issues. It is well known that damping and structural frequencies play a fundamental role in the structural response under wind loads; in fact, an increase of damping would lead to remarkable beneficial effects on the dynamic response of these structures. This paper focuses the attention on a mathematical model of a damped gyroscopic device conceived as a dynamic stabilizer for such slender structures; the proposed mechanical device allows for an increase of structural damping with a consequent improvement of dynamic performances of the tower subjected to wind loads. A parametric study for different mechanical properties of the gyroscopic device has been conducted in the present paper aiming to assess the effectiveness of the proposed apparatus for different configurations of the proposed device.
\end{abstract}

Keywords: Monopole towers, Slender structures, Gyroscopic stabilizer, Frequency increment, Damping increment. 


\section{INTRODUCTION}

The Monopole towers are slender structures usually employed for supporting communication devices or lighting poles. It is generally recognized that these structures are very sensitive to wind effects and to uncertainties related to wind forces and to structural dynamic features $[1,2]$. In past years, the authors have studied the dynamics of the monopole towers [3], among wind sensitive structures, ensuring the presence of specific design problems related to the definition of wind load and the dynamic characteristics of the structure as shown in $[4,5]$. Dynamic performances of these structures are therefore strongly affected by environmental conditions and by dynamic and geometrical properties of these structures $[6,7]$.

Proper assessment of environmental conditions (ground roughness, exposition, orographic placement) combined to dynamic structural characteristics of the mast, is an important topic since these factors deeply affect affects the structural dynamic behavior of these systems $[3,7$, 8]. However, despite the assessment of dynamic characteristics and the examination of the environmental conditions, cautious attention to fatigue problems has to be paid for this typology of slender structures e.g. [7-9].

Gyroscopic systems have been always studied in automotive and in mechanical systems [10-14]; moreover, the interaction between the beam and gyroscopic devices has been studied by several authors for examining their dynamic behavior [15-18] moreover recently gyroscopic devices were applied as structural isolation device in seismic engineering [19].

This paper proposes a direct integration approach for simulating the structural interaction between the gyroscopic device and the vertical mast of the tower, utilizing a mathematical model of the gyroscopic device applied to lumped mass mast model already developed by the author in a previous paper [20], in the present work, a parametric study performed on mechanical properties of the gyroscopic device has been performed, aiming to assesses and to optimizes the performances of the proposed device.

The principal component of the gyroscopic system is a rotating mass, having a horizontal angular momentum $\Omega$ perpendicular to the mast axis; the rotating mass is connected to the vertical shaft by a mechanical system provided with rotational damped spring system, which allows relative rotations (in the horizontal plane) between the vertical shaft and the rotating mass. The gyroscopic system reacts to the mast horizontal oscillations by coupling its vibrations with the relative rotation of the gyroscopic device, consequently transferring the energy from the mast to the damped gyroscopic device [11].

Results show that the gyroscopic stabilizer allows, for an increasing of the natural frequency of the system, as confirmed by Yamanaka [21] and Carta et al. [17, 19] and for a remarkable increasing of damping of the tower; which, plays an important role in the reduction of wind-induced vibrations amplitude of slender structures [22-24]. The study demonstrates that the effectiveness of the device depends on the mechanical properties of the gyroscopic device which have to be properly set in order to optimize the efficiency of the proposed apparatus.

\section{BACKGROUND MATHEMATICAL MODELS AND BENCHMARK SYSTEM}

The present approach is based on the dynamic equilibrium equations of the gyroscopic lumped system with gyricity $\boldsymbol{\Omega}$ described by D'Eleuterio [11] and on the hypothesis of the continuous Euler-Bernoulli beam dynamic equation; in the present work, the structural damping of the mast is not taken into account. The proposed formulation is valid for a gyroscopic device positioned at the elevation $H_{\Omega}$ of the mast, the equation considers the bending mo- 
ments $M_{u u}, M_{v v}$ and of the torsional moment $M_{w w}$ of the mast acting on the gyroscopic device. In case of small oscillations dynamic equation of the lumped gyroscopic device can be written as:

$$
\begin{gathered}
J_{\Omega, 11} \ddot{\alpha}+C_{\alpha} \dot{\alpha}+K_{\alpha} \alpha+M_{w w}^{d o}-M_{w w}^{u p}=-\Omega \dot{v}^{\prime} \\
J_{\Omega, 22} \ddot{v}^{\prime}+M_{u u}^{d o}-M_{u u}^{u p}=\Omega \dot{\alpha} \\
J_{\Omega, 33} \ddot{u}^{\prime}+M_{w w}^{d o}-M_{w w}^{u p}=0
\end{gathered}
$$

Where $u$ and $v$ respectively indicate displacements in the horizontal and transversal direction, the dot $\cdot$ indicates time derivative, the superscripts ' indicate derivative with respect to the vertical axis $w$ and the superscripts up and do indicate the position "up" and "down" of the cross-section respect to the position of the gyroscopic device. According to above-mentioned hypotheses and after a proper static matrix condensation dynamic equilibrium of the undamped free vibrations of the system can be written in matrix form as:

$$
\mathbf{M} \ddot{\mathbf{x}}+\mathbf{K x}=\mathbf{0}
$$

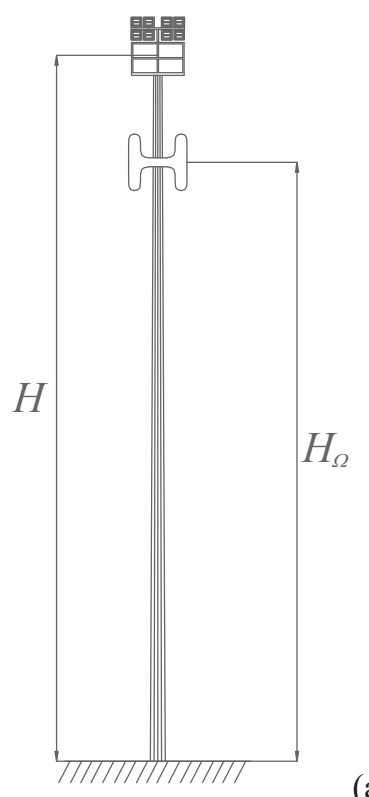

(a)

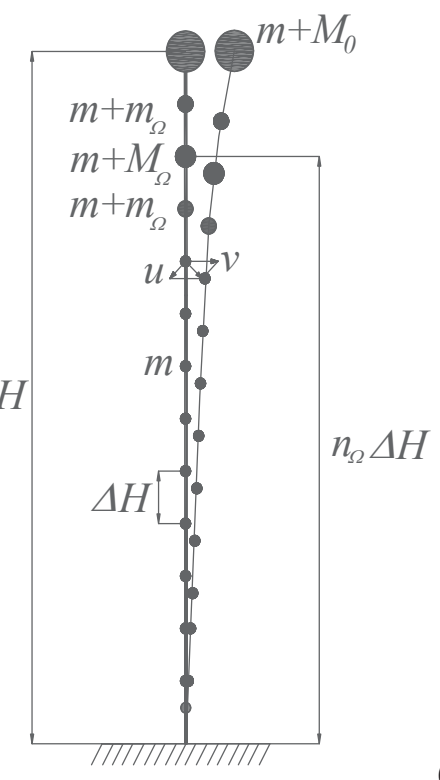

(b)

Figure 1: Monopole tower with gyroscopic device: (a) Schematic of the structure, (b) Generalized lumped mass model, orientation and displacements.

Where $\mathbf{M}$, and $\mathbf{K}$ represent respectively the $2 N \times 2 N$ mass and stiffness matrix of the system depicted in Fig.1 $\ddot{\mathbf{x}}$ and $\mathbf{x}$ symbolize respectively generalized accelerations and displacements vectors of the $2 N$ degrees of freedom (DOF) of the structure in the two directions $u$ and $v$, being $N$ the number of lumped masses of the system.

Accounting on the further DOF $\alpha$ of the gyroscopic device and according to above mentioned hypotheses Eq.1 and Eq.2 [20], can be conveniently combined as a function of a nondimensional time $\tau=\bar{\omega}_{0} t$ and converted to non-dimensional displacements $\chi_{j}={ }^{x_{j}} / \mathrm{D}$ in a matrix forma as:

$$
\mathbf{M}_{\mathrm{n}} \chi^{\prime \prime}+\frac{C_{\alpha}}{M_{0}} \mathbf{C}_{\mathrm{n}} \chi^{\prime}+\bar{\omega}_{0}^{2} \mathbf{K}_{\mathrm{n}} \chi=\mathbf{K}_{\mathrm{n}, \Omega}\left(\chi, \chi^{\prime}\right)
$$



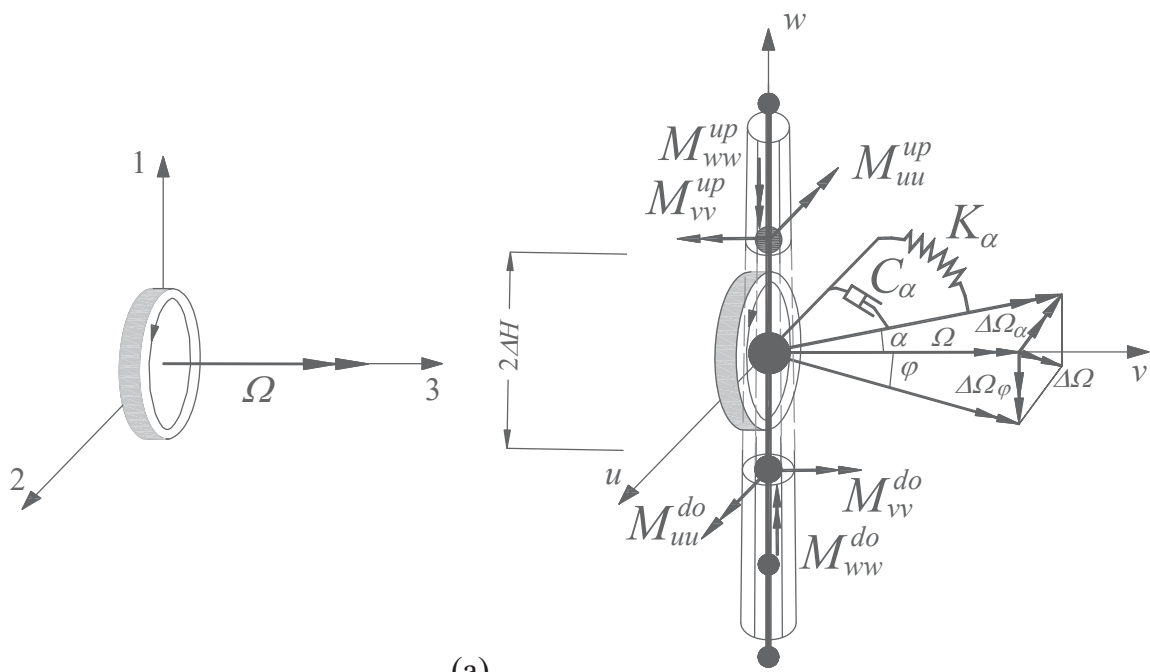

(a)

(b)

Figure 2: (a) Rotating mass with the local reference axes, (b) schematic of the gyroscopic device placed on the mast.

Where $\mathbf{M}_{\mathbf{n}}, \mathbf{C}_{\mathbf{n}}$ and $\mathbf{K}_{\mathbf{n}}$ represent respectively the normalized mass damping and stiffness $(N+1) \times(N+1)$ matrix of the system depicted in Fig.1, were the additional DOF present in the matrices is due to the further DOF $\alpha$ of the gyroscopic device and $\bar{\omega}_{0}=\sqrt{k_{0} / M_{0}}$ is the reference pulsation of a single DOF with reference mass $M_{0}$ and stiffness $k_{0}=3 E I / H^{3}, \mathbf{M}_{\mathbf{n}}=\mathrm{M} / M_{0}, \mathbf{C}_{\mathbf{n}}$ and $\mathbf{K}_{\mathbf{n}}=\mathrm{K} / M_{0}$ represents respectively the normalized mass matrix, damping matrix and stiffness matrix of the system. The quantities $\chi^{\prime \prime}, \chi^{\prime}$ and $\chi$ are respectively the unknown nondimensional vectors of the generalized accelerations, velocities and displacements with respect to the dimensionless time $\tau, C_{\alpha}=2 \bar{\omega}_{\alpha} J_{\alpha} \zeta_{\alpha}$ is the damping coefficient of the of the gyroscopic device with stiffness $K_{\alpha}$, inertia $J_{\alpha}=J_{\Omega, 11}$, undamped pulsation $\bar{\omega}_{\alpha}=\sqrt{k_{\alpha} / J_{\alpha}}$ and damping ratio $\zeta_{\alpha}$. Normalized undamped pulsation of the gyroscopic device can therefore written as function of the reference system pulsation $\bar{\omega}_{0}$ as $\alpha_{\alpha}={ }^{\omega_{\alpha}} / \bar{\omega}_{0}[20]$.

As it can be noticed form an examination of Eq. (1), displacements in the $u$-w plane do not involve the gyricity of the system, moreover, given the hypothesis of a lumped mass model having their barycenter coincident with the mast shear center cross-section; displacement $u$ (in the $u-w$ plane) can, therefore, considered decoupled from the remaining degrees of freedom of the structure [25]. The model described in section 2.1 with $2 \times(N+1)$ DOF, can be consequently reduced to a $N+1$ DOF system for proper boundary conditions which does not involve displacements in the $u$ - $w$ plane. Accounting of the above-mentioned hypotheses the lumped mass model with the gyroscopic device will be treated in the following as a $N+1$ DOF system.

To assess the effectiveness of the proposed apparatus, numerical integration of the discrete dynamic equations has been conducted, similar verification approaches were already utilized by the authors in [20, 26-28]. 


\section{NUMERICAL RESULTS}

The prototype of the proposed gyroscopic device applied to the lumped mass system depicted in Fig. 1 is examined in this section. Without any loss of generality, the lumped masses are constant and equally distributed in the shaft with $m_{i}=m$; in order to perform a parametric study, generalized properties of the system are defined as a function of the reference system having mass $M_{0}$ (mass of upper platform), stiffness $k_{0}$ (stiffness of a cantilever beam with height $H$ and flexural stiffness $E I$ ), reference pulsation $\bar{\omega}_{0}$.

The gyroscopic system has been placed in the $N$-1-th mass of the lumped mass model with $N=16$. The considered structure is a slender lighting pole typically utilized for illumination of large areas, sizes and structural properties are shown in Table 1:

\begin{tabular}{llllllllll}
\hline $\begin{array}{l}H \\
(\mathrm{~m})\end{array}$ & $\begin{array}{l}\rho_{\text {mast }} \\
(\mathrm{kg} / \mathrm{m})\end{array}$ & $\begin{array}{l}M_{0} \\
(\mathrm{~kg})\end{array}$ & $\begin{array}{l}D \\
(\mathrm{~mm})\end{array}$ & $\begin{array}{l}z_{\Omega} \\
(\mathrm{m})\end{array}$ & $\begin{array}{l}E I \\
\left(\mathrm{~N} \times \mathrm{m}^{2}\right)\end{array}$ & $\begin{array}{l}N \\
(-)\end{array}$ & $\begin{array}{l}\Delta z \\
(\mathrm{~m})\end{array}$ & $\begin{array}{l}m \\
(\mathrm{~kg})\end{array}$ & $\begin{array}{l}\bar{\omega}_{0} \\
(\mathrm{rad} / \mathrm{s})\end{array}$ \\
\hline 22 & 71.47 & 687.9 & 211.2 & 20.6 & $3.07 \mathrm{e}+7$ & 16 & 1.37 & 98.27 & 1.88 \\
\hline
\end{tabular}

Table 1: Structural properties of the monopole tower and of the corresponding lumped mass model.

Generalized properties of the gyroscopic system (refer to Table 2) are defined as a function of the structural characteristics of the mast, therefore non-dimensional magnitudes defining the gyroscopic device are: the mass ratio $\mu_{M, \Omega}=M_{\Omega, T O T} M_{0}$ (respect to the reference mass $M_{0}$ ), the radius of gyration ratio $\mu_{\rho, \Omega}=\rho_{\Omega} / D$ (respect to mast diameter $D$ ), so that $J_{\Omega, p}=M_{0} \mu_{M, \Omega}\left(D \mu_{\rho, \Omega}\right)$ and $J_{\Omega, 11}=J_{\Omega, 22}=0.5 J_{\Omega, p}$. Mechanical properties of the gyroscopic apparatus as stiffness $k_{\alpha}$, damping coefficient $C_{\alpha}$, damping ratio $\zeta_{\alpha}$, pulsation $\bar{\omega}_{\alpha}$ and normalized pulsation $\alpha_{\alpha}$ are illustrated in Table 2 .

\begin{tabular}{lllllllllllll}
\hline $\begin{array}{l}\mu_{M, \Omega} \\
(-)\end{array}$ & $\begin{array}{l}M_{\Omega, T O T} \\
(\mathrm{~kg})\end{array}$ & $\begin{array}{l}M_{\Omega} \\
(\mathrm{kg})\end{array}$ & $\begin{array}{l}m_{\Omega} \\
(\mathrm{kg})\end{array}$ & $\begin{array}{l}\mu_{\rho, \Omega} \\
(-)\end{array}$ & $\begin{array}{l}\rho_{\Omega} \\
(\mathrm{m})\end{array}$ & $\begin{array}{l}J_{\Omega, \rho} \\
\left(\mathrm{kg} \times \mathrm{m}^{2}\right)\end{array}$ & $\begin{array}{l}J_{\Omega, 11}=J_{\Omega, 22} \\
\left(\mathrm{~kg} \times \mathrm{m}^{2}\right)\end{array}$ & $\begin{array}{l}K_{\alpha} \\
(\mathrm{N} \times \mathrm{m})\end{array}$ & $\begin{array}{l}C_{\alpha} \\
(\mathrm{N} \times \mathrm{m} \times \mathrm{s})\end{array}$ & $\begin{array}{l}\zeta_{\alpha} \\
(-)\end{array}$ & $\begin{array}{l}\bar{\omega}_{\alpha} \\
(\mathrm{rad} / \mathrm{s})\end{array}$ & $\begin{array}{l}\alpha_{\alpha} \\
(-)\end{array}$ \\
\hline 0.10 & 68.79 & 67.15 & 0.82 & 1.42 & 0.30 & 6.14 & 3.07 & - & 11.66 & 10 & 0.19 & 0.10 \\
\hline
\end{tabular}

Table 2: Mechanical properties of the gyroscopic device.

It must be noted that the presence of the mass of the gyroscopic device affects the global behavior of the original structure, is therefore important to reduce as much as possible the additional mass of the gyroscopic device $M_{\Omega, T O T}$ and its radius of gyration $\rho_{\Omega}$ which respectively influence the structure natural frequency and its aerodynamic behavior. Therefore, careful attention must be paid for the design of the gyroscopic apparatus especially regarding the additional mass increment for possible buckling effects and frequency reduction and its size for a suitable aerodynamic design. Equation 3 has been solved by fourth-order Runge-Kutta algorithm [34] which requires appropriate initial boundary conditions at $\tau=0$ selected to examine the dynamic response of the first mode by imposing zero velocities and initial displacements in the $y-z$ plane, proportional to linear eigenvector $\Phi$ of the structure without gyricity $(\Omega=0)$ according to a preselected amplitude parameter $\delta=y / D$. 


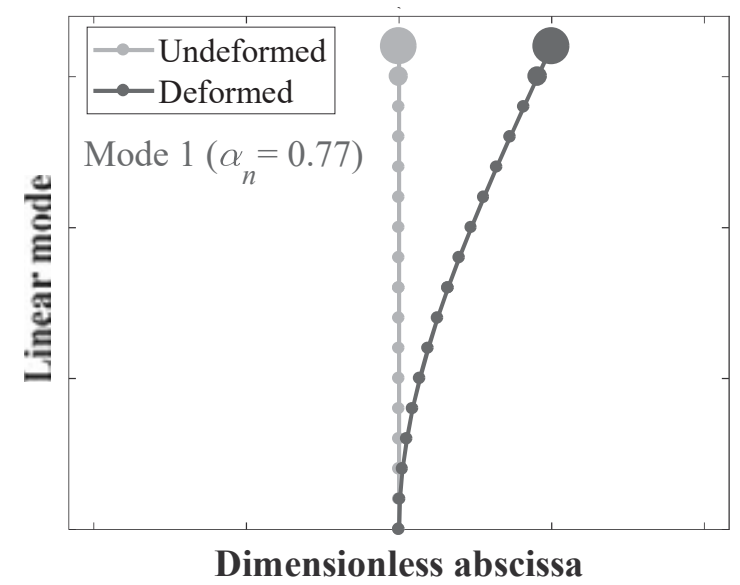

Fig. 3. First vibration mode of the examined lumped mass model for $\mu_{M, \Omega}=0.10$, without gyricity $(\Omega=0)$.

Free vibrations corresponding to the first mode of the dynamic system with the gyroscopic device are illustrated in Fig. 4, Fig. 4a shows normalized displacements $x / D$ of the top of the tower and angular rotations $\alpha$ of the gyroscopic device acting with gyricity $\Omega=\omega J_{\Omega, p}(\omega=180$ $\mathrm{rad} / \mathrm{sec}$ ) for different stiffness $K_{\alpha}$ of the spring device. Results illustrate the effectiveness of the proposed device in increasing the total damping of the mast, low values of stiffness $K_{\alpha}$ involve higher values of angular rotations $\alpha$ (refer to Fig. 4b), which in turn allow higher equivalent damping ratios $\zeta_{\Omega}$ of the tower. As can be observed by the inspection of Fig. 4a, the effectiveness of the device increases with the decreasing of the device spring $K_{\alpha}$, which allows (for a constant gyricity $\Omega$ ) the maximum effectiveness for $K_{\alpha}=0.01$, by an inspection of Fig. $4 \mathrm{~b}$, it must be noted that a further decreasing of the $K_{\alpha}$ would led, as expected, to unacceptable angular rotations which it would not satisfy the hypotheses of small oscillations on which Equation 1 is based.
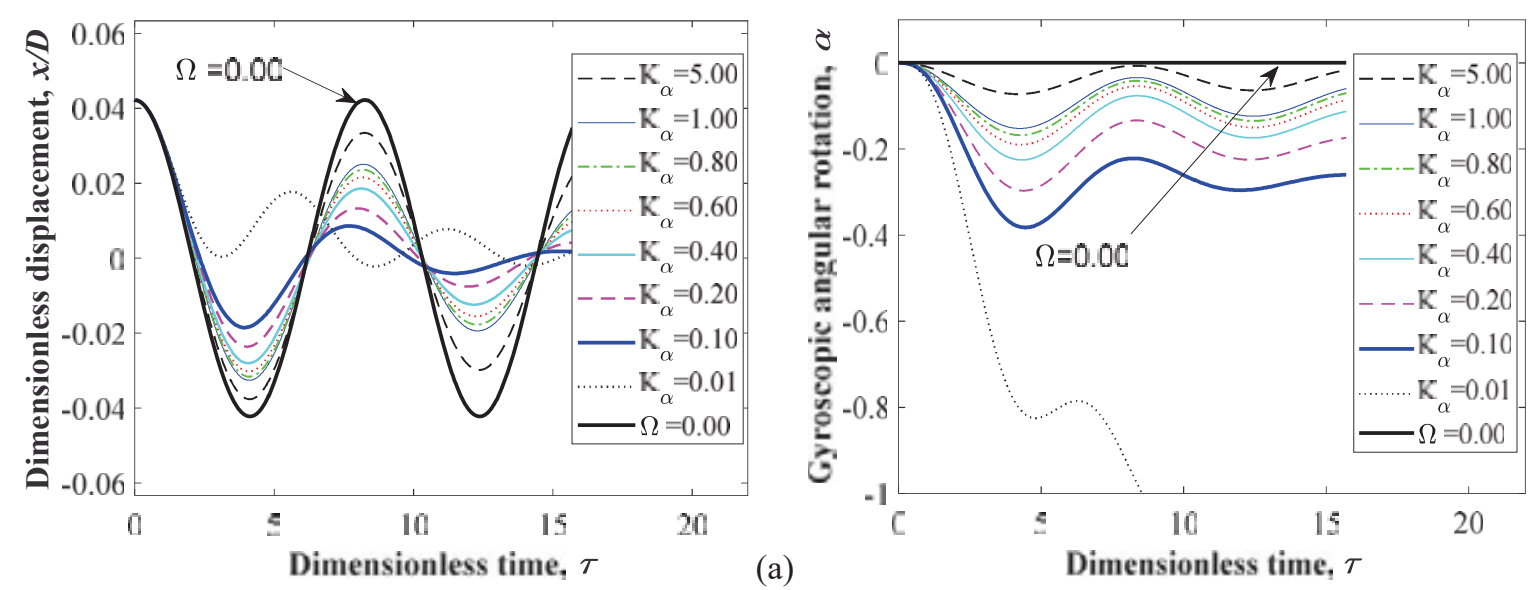

Figure 4. Time history analysis of the free vibrations of the first mode of the tower with $\mu_{M, \Omega}=0.10, \mu_{\rho, \Omega}=1.42$, corresponding to an amplitude parameter $\delta=y / D=0.20$ with constant gyricity $\Omega=\omega J_{\Omega, p}(\omega=180 \mathrm{rad} / \mathrm{sec})$ for different stiffness of the gyroscopic device spring $K_{\alpha}$ : (a) normalized horizontal displacements $y / D$ of the $N$-th point and (b) rotations $\alpha$ of the gyroscopic device.

The results of Fig.4 are corroborated by the data illustrated in Fig.5. which show an increasing of $\alpha_{\Omega}$ and of $\zeta_{\Omega}$ with the decreasing of the spring stiffness $K_{\alpha}$. The effectiveness of the proposed gyroscopic device acting with the same gyricity, is therefore directly connected to the spring stiffness $K_{\alpha}$. 

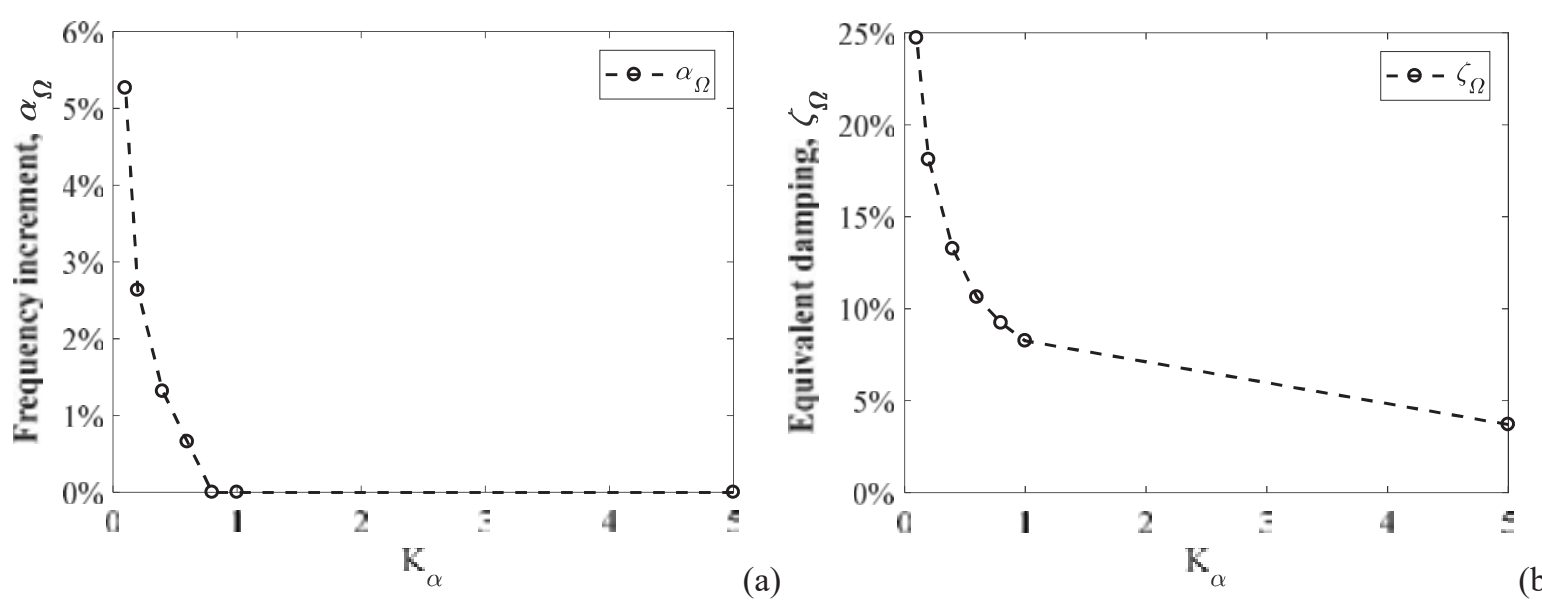

Figure 5. Parametric analysis as function of the stiffness of the gyroscopic device spring $K_{\alpha}$ for the first mode of the tower with $\mu_{M, \Omega}=0.10, \mu_{\rho, \Omega}=1.42, \delta=y / D=0.20$ and constant gyricity $\Omega=\omega J_{\Omega, p}(\omega=180 \mathrm{rad} / \mathrm{sec})$, (a) Normalized damped frequency $\alpha_{\Omega}$ and (b) equivalent damping $\zeta_{\Omega}$ of the tower.

\section{CONCLUSIONS}

This paper investigated the effects of a prototype of a gyroscopic device installed in slender monopole tower. Results show that the presence of the device allows for an increase of the frequency and for a remarkable increase of damping of the tower; the effectiveness of the device depends on its mechanical properties. A further parametric study assesses and optimizes the performances of the gyroscopic device as a function of the angular spring stiffness $K_{\alpha}$ which connects the gyroscope to the mast. Results illustrate that stiffness spring $K_{\alpha}$ can be considered a key parameter for adjusting and setting the mechanical properties of the gyroscopic device.

\section{REFERENCES}

[1] I. Venanzi, A.L. Materazzi, L. Ierimonti, Robust and realible optimization of windexcited cable stayed masts, Journal of Wind Engineering and Industrial Aerodynamics 147 (2015) 368-379.

[2] G. Solari, L.C. Pagnini, Gust buffeting and aeroelastic behaviour of poles and monotubular towers, Journal of Fluids and Structures 13(7-8) (1999) 877-905.

[3] G.F. Giaccu, L. Caracoglia, Wind-load fragility analysis of monopole towers by Layered Stochastic-Approximation-Monte-Carlo method, Engineering Structures 174 (2018) 462-477.

[4] M. Pavan Kumar, Effect of wind speed on structural behaviour of Monopole and selfsupport telecommunication towers, Asian Journal of Civil Engineering 18(6) (2017) 911927.

[5] U. Støttrup, Masts and towers, Journal of the International Association for Shell and Spatial Structures 55 (2014) 79-88.

[6] L. Pagnini, G. Solari, Gust buffeting and turbulence uncertainties, Journal of Wind Engineering and Industrial Aerodynamics 90(4) (2002) 441-459. 
[7] M.P. Repetto, G. Solari, Dynamic alongwind fatigue of slender vertical structures, Engineering Structures 23(12) (2001) 1622-1633.

[8] M.P. Repetto, G. Solari, Dynamic crosswind fatigue of slender vertical structures, Wind and Structures 5(6) (2002) 527-542.

[9] M.P. Repetto, G. Solari, Wind-induced fatigue of slender vertical structures, 11th International Conference on Wind Engineering, Wind Science and Engineering Research Center, Texas Tech University, Lubbock, Texas, USA, 2003, pp. 1803-1810.

[10] Y. Kan, Y. Psinh, A.-C. Lee, Investigation on the steady-state response of a symmetric rotors, Journal of Vibration and acoustics 114 (1992).

[11] G.M.T. D'Eleuterio, Dynamics of gyroelastic vehicles, Institute for aerospace studies, Toronto, 1986.

[12] M. Ghommema, A.H. Nayfeh, S. Choura, F. Najar, E.M. Abdel-Rahman, Modeling and performance study of a beam micro gyroscope, Journal of Sound and Vibration 329 (2010) 4970-4979.

[13] J. Li, D. Chen, G. Liu, Y. Li, B. Xu, Analysis of the gyroscopic effect on the hydroturbine generator unit, Mechanical Systems and Signal Processing 132 (2019) 138-152.

[14] R. Lot, J. Fleming, Gyroscopic stabilisers for powered two-wheeled vehicles, Vehicle System Dynamics 57(9) (2019) 1381-1406.

[15] A. Sinha, S. Bose, A. Nandi, S. Neogy, A precessing and nutating beam with a tip mass, Mechanics Research Communications 53 (2013) 75- 84.

[16] M. Esmaeilia, N. Jalilib, M. Duralia, Dynamic modeling and performance evaluation of a vibrating beam microgyroscope under general support motion, Journal of Sound and Vibration 301 (2007) 146-164.

[17] M. Garau, G. Carta, M.J. Nieves, I.S. Jones, N.V. Movchan, A.B. Movchan, Interfacial waveforms in chiral lattices with gyroscopic spinners, Proc. R. Soc.A 474 (2018) 20180132.

[18] M.J. Nieves, G. Carta, I.S. Jones, A.B. Movchan, N.V. Movchan, D.M. Sykes, Vibrations and elastic waves in chiral multi-structures, Journal of the Mechanics and Physics of Solids 121 (2018) 387-408.

[19] G. Carta, I.S. Jones, N.V. Movchan, A.B. Movchan, M.J. Nieves, Gyro-elastic beams for the vibration reduction of long flexural systems, Proceedings of the Royal Society A: Mathematical, Physical and Engineering Sciences 473 (2017) 1-17.

[20] G.F. Giaccu, Modeling a gyroscopic stabilizer for the improvement of the dynamic performances of slender monopole towers, Engineering Structures 215 (2020) 110607.

[21] K. Yamanaka, G. Heppler, K. Huseyin, Stability of gyroelastic beams, AIAA J. 34 (1996) 1270-1278.

[22] L.C. Pagnini, S. Lagomarsino, G. Solari, Experimental assessment of the damping of steel poles and monotubular towers (in Italian), "Costruzioni Metalliche", Journal of the Italian Technical Association for Steel Construction (CTA) LI(1) (1999) 39-51.

[23] G. Solari, Evaluation and role of damping and periods for the calculation of structural response under wind loads, Journal of Wind Engineering and Industrial Aerodynamics 59(2-3) (1996) 191-210.

[24] G. Solari, C. Pagnini, The action and effects of wind on poles and monotubular towers (in Italian), "Costruzioni Metalliche", Journal of the Italian Technical Association for Steel Construction (CTA) L(4) (1998) 29-51.

[25] C. Senthamaraikannan, R. Ramesh, Experimental investigation on vibration characteristics of woven carbon fabric-reinforced composite beams of various crosssectional shapes, Proceedings of the Institution of Mechanical Engineers Part L Journal of Materials Design and Applications 230(1) (2014) 64-74. 
[26] G.F. Giaccu, L. Caracoglia, B. Barbiellini, Modeling unilateral response in the crossties of a cable network: deterministic vibration, Journal of Sound and Vibration 333(19) (2014) 4427-4443.

[27] G.F. Giaccu, L. Caracoglia, Generalized power-law stiffness model for nonlinear dynamics of in-plane cable networks, Journal of Sound and Vibration 332(8) (2013) 19611981.

[28] G.F. Giaccu, L. Caracoglia, A displacement-based approach for determining nonlinear effects on pre-tensioned-cable cross-braced structures Journal of Sound and Vibration 394 (2017) 465-481. 